\title{
Gap Analysis of Annular Groove MR Brake
}

\author{
Jin Huang*, Zhen Qiao, Yi Zhou \\ ${ }^{1}$ College of Mechanical Engineering, Chongqing University of Technology, Chongqing 400054, China \\ *Corresponding author: jhuangcq@163.com
}

\begin{abstract}
Keywords: MR fluids, braking torque, design gap Analysis
Abstract: This paper provides an annular groove Magnetorheological(MR) brake. Bingham model is used to describe the constitutive behavior of MR fluids subject to an applied magnetic field. The theoretical analysis of the shear flow and braking torque of MR fluid between two parallel annular disks in annular groove MR brake is presented. The performance experimental system of brake is established to test the properties of annular groove MR brake. Based on the braking torque equation, the expression for the minimum active fluid volume and gap of MR fluid exposed to the magnetic field between two parallel annular disks is derived to provide the theoretical foundation for the key geometric design in annular groove MR brake. Based on the function of brake, the design elements and methods of an annular groove MR brake are established. The results indicate that the braking torque greatly influenced by the magnetic flux density. Under the premise of given braking torque, the speed and control torque ratio, a designer can select the MR fluids, calculate the effective gap and volume, draw the desired function diagram.
\end{abstract}

\section{Introduction}

MR brake is a device that provides the braking torque by the shear stress of the MR fluids. Based on the MR technology, the braking torque can be controlled by applied magnetic field. Much research has been devoted to working principle, performance and design methods of MR transmission. Huang [1] investigated the working principle of cylindrical MR brakes and the mechanical model of MR brake has been established to analyzes the torque transmission capability of brake. Ameen H. El-Sinawi[2] studied the modeling and control of MR damper. Song Chen [3] has analysis the relationships between shear yield stress, magnetic field, size, and volume fraction of MR fluid in two parallel discs are established based on the chain structure of magnetic particle under applied magnetic field. Using magnetically conductive and non-conductive rings a serpentine flux path has been developed to weave the magnetic flux through the MRF by Doruk Senkal [4]. Tran Hai NAM [5] has proposed a new approach to increase the weak force in shear mode by the application of a large size magnetic particle which is a small steel roller. Kerem Karakoc [6] reported the method for designing the automotive MR brake. Jin Huang [7] analyzed the MR shear transmission driven by shape memory alloy spring. The MR brake and MR clutch have been studied by many investigators in recent years. However, few studies have been reported on the effective volume and effective working gap of annular groove MR brake. Based on the rheological properties of MR fluid, the working principle of annular groove MR brake was proposed in this study. This study aims to establish the key geometric of annular groove working gap in MR brake based on flow and transmission equation of MR fluid. Based on the brake function, the design method of the annular groove MR brake is established.

\section{Braking torque}

\section{Operational principle}

The schematic of the annular groove MR brake is shown as Fig.1. The MR fluid is filled with the working gap between the fixed disk and the Rotating disk in the brake. MR fluid is formed like a ring during operation of brake, the surface of the ring perpendicular to the axis of rotation. In the absence of an applied magnetic field, MR fluid flow freely and exhibit Newtonian-like fluid behavior. The torque 
transmitted by the viscous stress of the MR fluid in the brake is much smaller so that the suspended particles of the MR fluid cannot restrict the rotating disk motion of the brake. However, in the course of operation, a magnetic flux path is formed when electric current is put through the coil-assembly. As a result, the MR particles are gathered to form chain-like structures, in the direction of the magnetic flux path. These chain-like structures increase the shear stress of the MR fluid. With the increase of the applied magnetic field, the shear stress and the torque developed by the MR fluid go up rapidly. The amount of torque can be adjusted continuously by changing the input current.

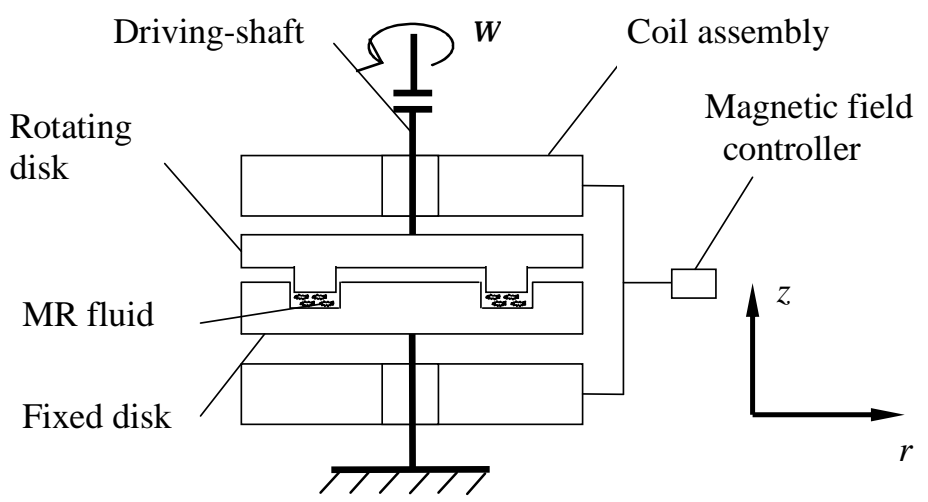

Fig.1 Schematic of the annular groove MR brake

\section{Torque Analysis}

In order to derive the equation of the fluid flow in the gap between two parallel circular discs, the following assumptions are given: the fluid is incompressible. There is no flow in radial direction and axial direction, but only tangential flow. The flow velocity of MR fluid is a function of radius. The pressure in the thickness direction of MR fluid is constant. The strength of magnetic field in the gap of the activation region is well-distributed. In cylindrical coordinates $(r, \theta, z)$, the distribution of the flow velocity is

$$
V_{r}=0, V_{\theta}=r \omega(z), V_{z}=0
$$

Where $V_{r}, V_{\theta}$ and $V_{z}$ are the flow velocity of the fluid in the $r$-direction, the $\theta$-direction and $z$-direction, respectively, $\omega(z)$ is the rotation angular velocity of the fluid in the $\theta$-direction. The angular velocity $\omega(z)$ is the function of $z$-coordinate. The flow equation of the MR fluid in the $\theta$-direction may be approximated by

$$
\frac{d^{2} \omega(z)}{d z^{2}}=\frac{1}{\eta} \frac{d \sigma_{\theta \theta}}{d \theta}
$$

where $\frac{d \sigma_{\theta \theta}}{d \theta}$ is the gradient of the pressure in the $\theta$-direction, $\eta$ is the viscosity of MR fluid with no applied magnetic field. It is assumed that the fluid between two parallel circular discs is a steady-state flow, $\frac{d \sigma_{\theta \theta}}{d \theta}=$ const .

Integrating the equation (2), the rotation angular velocity $\omega(z)$ can be indicated as: 


$$
\omega(z)=\frac{z^{2}}{2 \eta} \frac{d \sigma_{\theta \theta}}{d \theta}+c_{1} z+c_{2}
$$

where $c_{1}$ and $c_{2}$ are the two integrating constant. It is assumed that the fluid in touch with the surface of the rotating disk has the same velocity as the rotating disk. The angular velocity of the MR fluid increases with z-direction. It is assumed that there is no magnetic flux line at the upper $\left(r=R_{2}\right)$ and the lower $\left(r=R_{1}\right)$ portions of the domain. So, applying the boundary conditions of $\omega(z)=\omega$ at $\mathrm{z}=h$ and $\omega(z)=0$ at $z=0$, the flow velocity $\omega(z)$ can be mathematically manipulated to yield the flow as follows:

$$
\omega(z)=\frac{\omega z}{h}+\frac{1}{2 \eta} \frac{\mathrm{d} \sigma_{\theta \theta}}{\mathrm{d} \theta}\left(z^{2}-z h\right)
$$

where $h$ is the gap between two parallel circular discs. $\omega$ is the rotational velocities of the rotating disk.

Upon application of a magnetic flied, MR fluids exhibit a Bingham plastic behavior. In this model, the flow of MR fluid is governed by Bingham's equations [1]:

$$
\tau=\tau_{y}(H)+\eta \hat{\mathcal{\gamma}}, \tau \geq \tau_{y}(H)
$$

where $\tau$ is the shear stress developed by MR fluid, $\hat{\gamma}$ is the fluid shear strain rate and $\tau_{y}(H)$ is the dynamic yield stress developed in response to an applied magnetic field $H$. Bingham model shows that without the applied magnetic field the yield stress of material is equal to zero, and the MR fluid exhibits the characteristics of a conventional Newtonian fluid. When the magnetic field is applied, the MR fluid exhibits the viscoplastic characteristics. For the flow field at $\tau \geq \tau_{y}(H)$, MR fluid flows with a viscous $\eta$. For the flow field at $\tau \leq \tau_{y}(H)$, the MR material behaves like a solid [8]. The rheological characteristics of MR fluid can be used in brake devices for the controlled flow of the fluid between two surfaces, which move in relation to each other.

It is assumed that the MR fluid between two parallel circular discs is continuous flow. Because the two discs in the brake are parallel each other, so the gradient's value of the pressure in the $\theta$-direction is zero $\left(\frac{d \sigma_{\theta \theta}}{d \theta}=0\right)$.The fluid shear strain rate of Eq.(5) may be approximated by

$$
\gamma=r \frac{\mathrm{d} \omega(z)}{\mathrm{d} z}
$$

The torque transmitted by the annular groove MR brake is calculated by integrating the shear stress of the MR fluid, as follows:

$$
T=2 \pi \int_{\mathrm{R}_{1}}^{R_{2}} \tau r^{2} d r
$$

where $R_{1}$ and $R_{2}$ are the actual inner and outer radius of the rotating disk in the MR fluid exposed to the magnetic field, respectively. Eqs.(4), (5), (6) and (7) can be mathematically manipulated to yield the torque as follows: 


$$
T=\frac{2 \pi}{3}\left(R_{2}^{3}-R_{1}^{3}\right) \tau_{y}(H)+\frac{\pi}{2 h}\left(R_{2}^{4}-R_{1}^{4}\right) \omega \eta
$$

Eq.(8a) suitable for MR transmition under application of weak and moderate magnetic field strength. When exposed to a strong magnetic field, the columnar structures of MR particles resulting in reduced effective radius of working gap in annular groove MR brake. Upon application of a strong magnetic field, the braking torque can be revised as

$$
T=\frac{2 \pi}{3}\left(R_{e 2}^{3}-R_{e 1}^{3}\right) \tau_{y}(H)+\frac{\pi}{2 h}\left(R_{2}^{4}-R_{1}^{4}\right) \omega \eta
$$

where $R_{e 1}$ and $R_{e 2}$ are the effective inner and outer radius of the MR fluid exposed to the magnetic field, respectively.

\section{Key geometric dimensions}

The minimum active fluid gap $h_{e}$ and effective diameter of working gap are key geometric dimensions of annular groove MR brake. Eq.(8b) shows that the maximum braking torque of annular groove MR brake consists of two parts when magnetic reaches saturation. The two parts of maximum torque developed by MR fluid are

$$
T_{H \max }=\frac{2 \pi}{3}\left(R_{e 2}^{3}-R_{e 1}^{3}\right) \tau_{H \max }
$$

$$
T_{\eta \max }=\frac{\pi}{2 h}\left(R_{2}^{4}-R_{1}^{4}\right) \omega \eta
$$

where $\tau_{H \text { max }}$ is maximum yield stress, $T_{H \text { max }}$ is the maximum yield torque developed by the yield stress and the $T_{\eta \max }$ is the maximum viscous torque developed by the viscosity of MR fluids.Eqs.(8) and (9) can be manipulated algebraically to derive the required minimum operational gap between two circular discs as follows:

$$
h_{e}=\frac{3}{4}\left(\frac{\eta}{\tau_{H \max }}\right)\left(\frac{R_{2}^{4}-R_{1}^{4}}{R_{e 2}^{3}-R_{e 1}^{3}}\right) \lambda \omega
$$

Where $\lambda$ denotes the desired control torque ratio, $\lambda=T_{H \max } / T_{\eta \max }$. Eq.(10) provides geometric constraints for a circular disc MR brake based on MR fluid properties $\left(\eta / \tau_{H \max }\right)$, the desired control torque ratio $\lambda$, and the rotational velocity of the brake $\omega$.

The necessary active volume induced by MR effect between two parallel discs is

$$
V_{e}=\pi h_{e}\left(R_{e 2}^{2}-R_{e 1}^{2}\right)
$$

(11)

The necessary active volume of MR fluid between two parallel discs of the brake can be obtained from Eqs. (9), (10) and (11) as: 
$V_{e}=k\left(\frac{\eta}{\tau_{H \max }^{2}}\right)\left(\frac{\lambda^{2}}{1+\lambda}\right) P_{M}$

Where, $k=\frac{9\left(R_{2}^{4}-R_{1}^{4}\right)\left(R_{2}^{2}-R_{1}^{2}\right)}{8\left(R_{e 2}^{3}-R_{e 1}^{3}\right)^{2}}, \lambda$ is control torque ratio, $\lambda=\frac{T_{H \max }}{T_{\eta \max }}, P_{M}$ is the transmission power of brake, $P_{M}=T_{\max } \omega$.

\section{Conclusions}

(1) Based on the rheological properties of MR fluid, the shear flow and braking torque of MR fluid between two parallel annular disks in annular groove MR brake is analyzed. The performance experimental system of brake is established to test the properties of annular groove MR brake. The braking torque is increased with the increase of magnetic flux density.

(2) The expression for the minimum active gap of MR fluid exposed to the magnetic field between two parallel annular disks is derived. The necessary active volume of MR fluid between two parallel discs of the brake in case of certain braking torque is obtained.

(3) The design elements and methods of an annular groove MR brake are established to analyses the anticipated performance of annular groove MR brake.

\section{Acknowledgments}

This work was supported by the National Natural Science Foundation of China(51175532 and 11272368) and by Scientific and Technological Research Program of Chongqing Municipal Education Commission (Grant No. KJ1400934).

\section{References}

[1] Huang J, Zhang J Q, Yang Y, "Analysis and design of a cylindrical magneto-rheological fluid brake", Journal of Materials Processing Technology, vol.129,no.1-3,pp.559-562,2002.

[2] Ameen H. El-Sinawi, Mohammad H. AlHamaydeh, and Ali A. Jhemi, "Optimal Control of Magnetorheological Fluid Dampers for Seismic Isolation of Structures", Mathematical Problems in Engineering, Volume. 2013, Article ID 251935, 7 pages, 2013. doi:10.1155/2013/251935.

[3] Song Chen, Jin Huang, Hongyu Shu, Tiger Sun, and Kailin Jian, "Analysis and Testing of Chain Characteristics and Rheological Properties for Magnetorheological Fluid," Advances in Materials Science and Engineering, Volume. 2013, Article ID 290691, 6 pages, 2013. doi:10.1155/2013/290691.

[4] Doruk Senkal, Hakan Gurocak, "Serpentine flux path for high torque MRF brakes in haptics applications", Mechatronics, vol.120,no.20,pp.377-383,2010.

[5] Tran Hai NAM, Kyoung Kwan AHN, "New approach to designing an MR brake using a small steel roller and MR fluid", Journal of Mechanical Science and Technology, vol.123,no.23,pp.1911- 1923,2009.

[6] Kerem Karakoc, Edward J. Park, Afzal Suleman., "Design considerations for an automotive magnetorheological brake", Mechatronics, vol.18,no.18,pp.434-447,2008.

[7] Jin Huang, Xu Chen, and Lirong Zhong, "Analysis and Testing of MR Shear Transmission Driven by SMA Spring", Advances in Materials Science and Engineering,Volume. 2013, Article ID 307207, 6 pages, 2013. doi:10.1155/2013/307207.

[8] Li W H, Du H, Guo N Q., "Dynamic behavior of MR suspensions at moderate flux densities", Materials Science and Engineering: A, vol.371,no.1-2,pp.9-15,2004. 